\title{
Visualization of Multiscale Dynamics of Hydrous Cold Plumes at Subduction Zones
}

\author{
Maxwell L. Rudolph, ${ }^{1}$ Taras V. Gerya, ${ }^{2}$ David A. Yuen, ${ }^{3,4}$ and Sara DeRosier ${ }^{3,4}$
}

1. Department of Geology, Oberlin College, Oberlin, $\mathrm{OH} 44074$, maxwell.rudolph@oberlin.edu

2. Institute of Geology, Mineralogy and Geophysics, Ruhr-University of Bochum, Universitaetstrasse 150, 44780 Bochum, Germany

3. Department of Geology and Geophysics, University of Minnesota, Minneapolis, MN 55455

4. Minnesota Supercomputing Institute, University of Minnesota, Minneapolis, MN 55455

\begin{abstract}
Recent increases in the computational power of high-performance computing systems have led to a large gap between the high-resolution runs of numerical simulations - typically approaching 50 to 100 million tracers and 1 to 5 million grid points - and the modest resolution of one to two million pixels for conventional display devices. This technical problem is further compounded by the variety of fields produced by numerical simulations and the limited bandwidth available through the Internet in the course of collaborative ventures. We have developed a visualization system using the paradigm of web-based inquiry to address these mounting problems. We have employed as a case study a problem involving two-dimensional multiscale dynamics of hydrous cold plumes at subduction zones. A Lagrangian marker method, in which the number of markers varies dynamically, is used to delineate the many different fields, such as temperature, viscosity, strain, and chemical composition. We found commercially available software to be insufficient for our visualization needs and so we were driven to develop a new set of tools tailored to high-resolution, multi-aspect, multiscale simulations, and adaptable to many other applications in which large datasets involving tens of millions of tracers with many different fields are prevalent. In order to address this gap in visualization techniques, we have developed solutions for remote visualization and for local visualization. Our remote visualization solution is a web-based, zoomable image service (WEB-IS) that requires minimal bandwidth while allowing the user to explore our data through time, across many thermo-physical properties, and through different spatial scales. For local visualization, we found it optimal to use bandwidth-
\end{abstract}


intensive, high-resolution display walls for performing parallel visualization in order to best comprehend the causal and temporal relationships between the multiple physical and chemical properties in a simulation.

\section{1) Introduction}

The dynamics of subduction is an important but intrinsically complicated problem in the geological sciences (Marsh, 1979; Stern, 2002). Subducting slab regions under volcanic arcs are characterized by intense seismic and volcanic activity, various phase transformations inside the slab, fluid and melt transport, and development of multiscale, buoyant, thermal-chemical features in the mantle wedge related to hydration and melting on the top of the subducting slab (Marsh, 1979; Davies and Stevenson, 1992; Tamura et al., 2002; Hall and Kincaid, 2001; Kincaid and Hall, 2003; Stern, 2002; Gerya and Yuen, 2003a; Peacock, 2003).

It is commonly thought (e.g., Tamura et al., 2002) hot rising mantle flows prevail in the mantle wedge above the subducting slab. However, hydration and partial melting along the slab can create a situation in which a Rayleigh-Taylor instability can develop at the top of a cold subducting slab within the relatively cold area characterized by an inverted temperature gradient. This can create paradoxically interesting geological phenomenon in which rising hydrous diapiric structures, colder than the asthenosphere by 300 to 400 degrees, are driven upward by compositional buoyancy (Tamura, 1994; Gerya and Yuen, 2003a). These "wet cold plumes" with a compositional, hydrous origin, are lubricated by viscous heating, have an upward velocity in excess of 10-100 cm/yr and penetrate the relatively hot asthenosphere in the mantle wedge within a couple of hundred thousand years (Gerya and Yuen, 2003a; Gerya et al., 2004). These plumes are fueled by partial melting of the hydrated mantle and subducted oceanic crust due to fluid release from dehydration reactions within the slab, including the decomposition of serpentine. Heating and decompression melting of these "hydrous cold plumes" may form incipient magma chambers under volcanic arcs (Gerya et al., 2004).

To model these processes, we have developed a coupled thermal-chemical dynamical 2-D model that accounts for the combined effects of both hydration and partial melting in the thermal, chemical, mechanical, rheological, and petrological domains (Marsh, 1979; Stern, 2002; Peacock, 2003). Recent advances in shared-memory supercomputer technology have enabled us to use large numbers $\left(10^{8}\right)$ of markers with this model, allowing high-resolution modelling with 
complex geometry (e.g., Ten et al., 1999). Recent innovations in the machine architecture on the IBM zSeries, Cray X1, SGI Altix 3000, and Japanese NEC supercomputers have placed at least $32 \mathrm{~GB}$ of memory on a single node, and the next generation of these machines will offer 64 to $128 \mathrm{~GB}$ on a single node. The anticipation of these coming technological innovations has figured prominently in our computational and visualization strategy. Combining conceptual, computational, and visualization advances, we describe a new technique for the visualization of a 2-D numerical model of a subducting slab region that exhibits multiscale thermomechanical and petrological features with a spatial resolution of a few tens of meters to 100 meters.

\section{2) Two-dimensional Numerical Model of the Slab Region}

We have used a regional 2-D numerical model (Fig. 1) for the upper mantle with a kinematically prescribed velocity boundary condition for the subducting slab and a simple model of hydration of the mantle wedge (Gerya et al., 2002, 2004; Gerya and Yuen, 2003a). The boundary conditions correspond to the corner flow model (Tovish et al., 1978) that accounts for asthenospheric mantle flow under the overriding plate. The initial position of the subduction zone is prescribed by a weak, $8 \mathrm{~km}$ thick, hydrated peridotite layer. During subduction, this layer is spontaneously substituted by weak, subducted crustal rocks and hydrated mantle, causing a decoupling along the plate interface. The top surface is calculated dynamically as a free surface using an $8 \mathrm{~km}$ thick top layer with a lower viscosity and density, simulating both the ambient atmosphere and seawater. To simulate incoming and outgoing flows of materials, recycling and addition of markers is organized along the three open boundaries of the model. A peculiar point worth noting about this procedure is the gradual increase (up to a factor of two) in the number of markers within the model with the progress of calculation. This particularly increases the density of the marker grid above the slab, which allows resolution of buoyant features within the wedge in very great detail, to about 20-40 meters.

Assuming continuous dehydration of the subducting slab, we account for hydration in the deforming mantle wedge with a vertically displaced hydration front (Fig. 1) relative to the top of the subducting slab (Gerya et al., 2002; Gerya and Yuen, 2003a). The hydration and related partial melting lead to a sharp decrease in the density and viscosity of mantle rocks, creating very favorable conditions for the development of Rayleigh-Taylor instabilities along the hydration front propagating from the slab. We have employed a composite, realistic rheology, which 
encompasses the variability of pressure, temperature, strain rate, degree of melting, and the many lithological phases (Gerya and Yuen, 2003a).

We have solved the momentum, continuity, and temperature equations for the 2-D creeping flow approximation and have considered both thermal and chemical buoyancies along with mechanical heating from adiabatic work and viscous dissipation. We have employed a recently developed code, I2VIS (Gerya and Yuen, 2003b), based on finite-differences with a non-diffusive marker-in-cell technique. This code can accurately model subduction on a fully staggered, rectangular, Eulerian grid for multiphase, viscoplastic flows of eight to twenty chemical components, and can account for the variable P-T dependent thermal conductivity and density, P$\mathrm{T}$, and strain-rate dependent rheology. Altogether, we have carried out around 50 high-resolution numerical runs with various subduction rates, slab ages, and numbers of markers. This code with a capability of incorporating 50 to 150 million markers allows us to resolve very fine details with an unprecedented spatial accuracy of between 25 to 100 meters, which is necessary to capture multiscale thermal-chemical-rheological features.

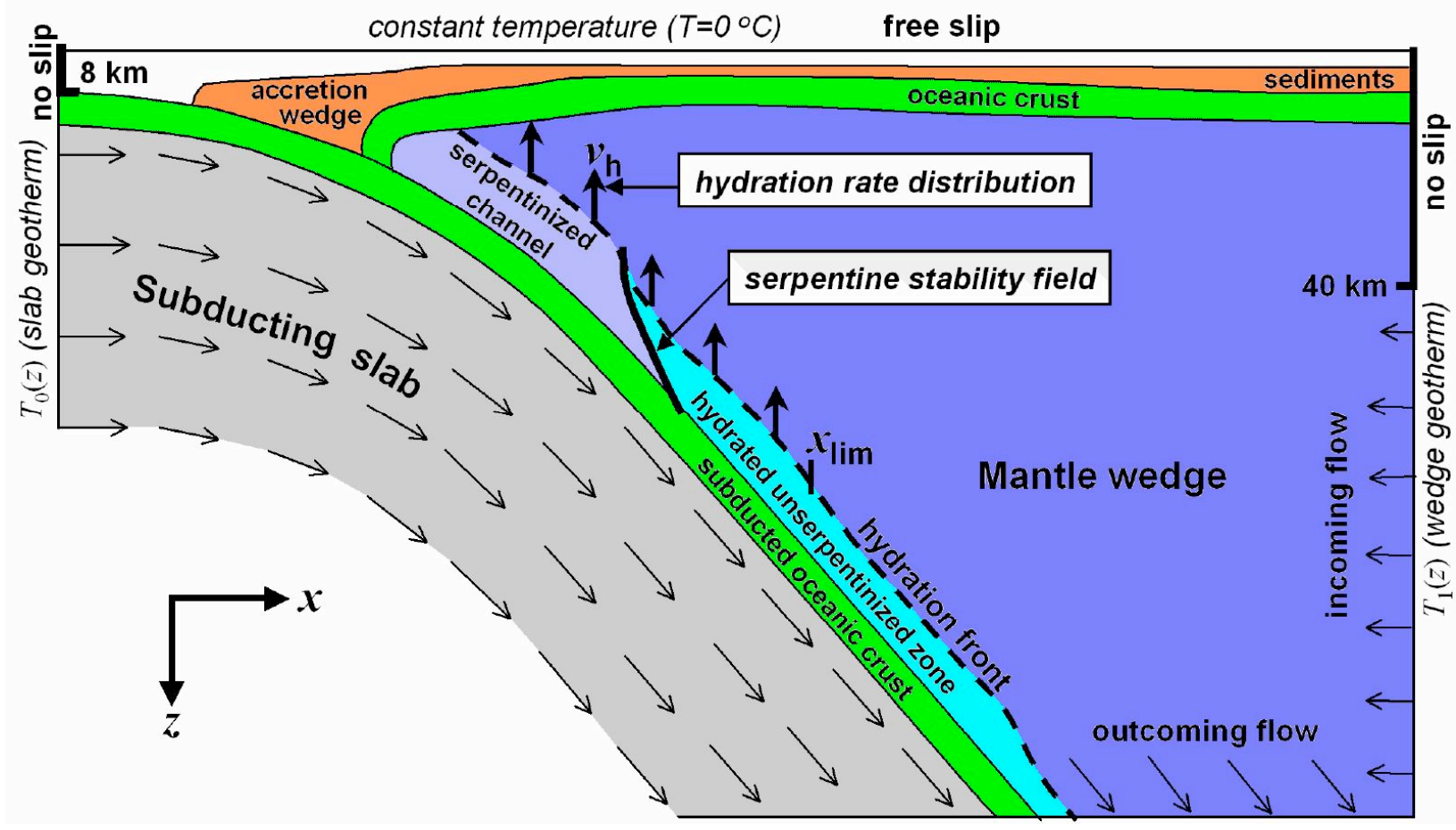

Figure 1. Numerical upper-mantle model designed for our two-dimensional numerical experiments. An intermediate stage of calculation with well-developed hydrated mantle zone is shown. The hydrated mantle is subdivided into two parts: an upper, serpentinized subduction channel developing within an upper, colder lithospheric portion of the wedge and a lower hydrated serpentine-free peridotite zone developing within a 
lower, hotter asthenospheric portion outside of the P-T stability field of serpentine (Schmidt and Poli, 1998). Initial conditions for calculation (Gerya and Yuen, 2003a) are taken as follows: the initial position of the subduction zone is prescribed by a weak, 8-km-thick, hydrated peridotite layer; initial temperature field in subducting plate is defined by an oceanic geotherm $\mathrm{T}_{0}(\mathrm{z})$ with a specified age; initial temperature distribution in overriding plate $\mathrm{T}_{1}(\mathrm{z})$ corresponds to equilibrium thermal profile with $0{ }^{\circ} \mathrm{C}$ at surface and $1350{ }^{\circ} \mathrm{C}$ at $32 \mathrm{~km}$ depth; initial structure of 8-km-thick oceanic crust is taken as follows (from top to bottom): sedimentary rocks $=1 \mathrm{~km}$, basaltic layer $=2 \mathrm{~km}$, gabbroic layer $=5 \mathrm{~km}$. Serpentine stability field is taken according to the experimental data for antigorite (Schmidt and Poli, 1998). Further details of model design and limitations are given by (Gerya and Yuen, 2003a).

\section{3) Temporal Evolution of Multiscale Features in Numerical Experiments}

One of our reference models demonstrates the development of multiscale phenomena that require new visualization techniques (Fig. 2). This reference run with 15 million tracers begins with a subduction angle of 45 degrees and a rate of $2 \mathrm{~cm} /$ year. After 10 to $17 \mathrm{Myr}$, a wave-like Rayleigh-Taylor instability emerges along the upper surface of the serpentine-free, hydrated peridotite zone. This buoyancy-driven instability is caused by the strong density contrast (100$200 \mathrm{~kg} / \mathrm{m}^{3}$ ) between the hydrated, lower density peridotite mixed with partially molten, subducted oceanic crust and the higher density of the overriding dry asthenosphere (Hall and Kincaid, 2001).

The density contrast competes successfully with a significant temperature contrast (300$500^{\circ} \mathrm{C}$, Fig. 2-middle column) between the hydrated and dry mantle. In addition, the dynamic viscosity of the hydrated mantle wedge is several orders of magnitude lower than that of the subducting slab and overriding dry mantle (Fig. 2-right column) according to wet and dry olivine flow laws (Ranalli, 1995). These contrasts create favorable conditions for the rapid escape of buoyant, wavelike features upward along the subducting plate within the partially molten lowviscosity peridotite zone.

The shape and internal geometry of the wave-like structures are very complex, reflecting the internal deformation and rotation of rocks within these waves during propagation (Fig. 2 at 11.9 Myr). The backward velocity of waves may be very high and often exceeds the subduction speed. Merging of several wavelike structures rising with different velocities commonly occurs. This causes the growth of rapidly propagating, relatively cold diapiric and finger-like structures 
("hydrous cold plumes") at a shallow level (Fig. 2 at 12.6 Myr), thus giving rise to the formation of an incipient magma chamber (Gerya et al., 2004).

\section{4) Visualization Challenges for High Resolution Numerical Experiments}

From Fig. 2 we can see that we must employ at least 50 million or more markers in order to unveil all of the possible complicated multiscale features in realistic subduction numerical experiments in which the resolution of around 50 meters is required. However, as the number of markers used in a marker-based simulation increases, distinct problems emerge in the visualization of this massive data set. Currently, there is an expanding gap between the ultra-high spatial resolution of the numerical simulations and the available resolution of the visualization display device. This disparity will only grow in the future as larger hybrid distributed/shared memory supercomputers become more widespread. 


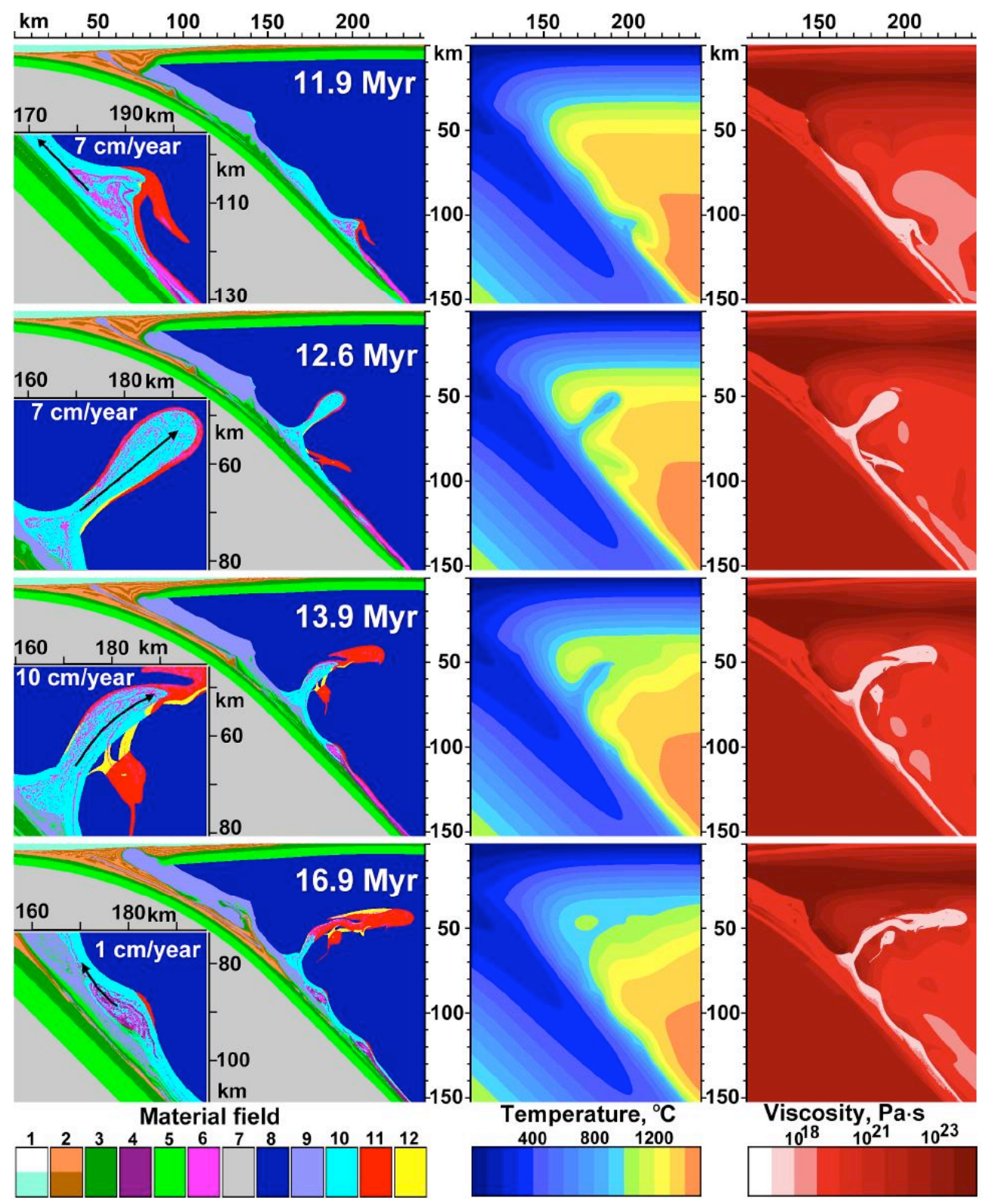

Figure 2. Dynamics of development of incipient magma chamber under the oceanic arc during early (12-17 Myr) stages of the ongoing subduction. ). 15 million markers are used in this model to represent the chemical composition (lithological) field with resolution about 100x100 m. Three columns show temporal evolution of the chemical composition (lithological) field (left column), temperature distribution (middle column) and viscosity field (right column) of the mantle wedge. The inset separate sketches represent enlarged $240 \mathrm{~km} \times 150 \mathrm{~km}$ areas of the original $400 \mathrm{~km} \times 200 \mathrm{~km}$ model. Zoomed areas in the left column show close-up view of selected wave-like ("cold waves") and diapiric structures ("hydrous cold plumes") developed in the numerical simulations. Left: Evolution of the distribution of rock types (color code): $1=$ weak layer on the top of the model (air, sea water), $2=$ sedimentary rocks; 3 and $4=$ solid and partially molten basaltic oceanic crust, respectively, 5 and $6=$ solid and partially molten gabbroic oceanic crust, respectively, $7=$ subducting mantle lithosphere, $8=$ unhydrated mantle wedge, $9=$ serpentinized mantle, 10 
$=$ hydrated unserpentinized (i.e. beyond the stability field of serpentine) mantle; $11=$ partially molten hydrated mantle; 12 = mantle quenched after partial melting.

By now, the resolution of our simulations has reached 500 million markers (Fig. 3), each of which carry at least ten different field attributes. Due to the discrete nature of the marker-based approach (Hockney and Eastwood, 1981), marker density is not completely homogeneous over the entire domain of interest. Consequently, the number of pixels required to view the entire model in full detail is actually greater than the number of markers involved. In areas with maximal marker density, a one-to-one mapping onto pixels is necessary for full resolution. This necessitates interpolation between markers in areas of lower marker density. The net result is that a single image from one of our simulations must be composed of somewhat more pixels than there are markers in the sumulation. Additionally, as many as 100 million markers have been used in some of our runs, and the number of markers is growing with each new simulation. For faster subducting slabs with $10 \mathrm{~cm} /$ year where more markers are needed to obtain sound results, we anticipate using at least 1 billion markers.

We have developed a new method for interpolation that produces adequate results for our purposes. It should be noted that the primary need for interpolation arises from the fact that it is more visually intuitive to interpret a continuous image than for the mind to fill in small holes in an image. In Fig. 4, we show our interpolation strategy, which requires that we employ searches for a specific signature in our data files. We feel that our present approach, based on linear and nearest-neighbor interpolation techniques, is best suited to its purpose since we need a fast response time. More sophisticated mathematical methods - such as the use of Voronoi cells (Sapiro, 2001) or level set methods (Osher and Fedkiw, 2002) - would require much greater computational costs, although we are considering implementing such a system in the future.

The massive number of pixels required to visualize the data from our simulations far exceeds the number of pixels on any readily available display device. A typical computer monitor contains 1.3 to 1.9 million pixels. A high-end CRT display can contain 3.3 million pixels (http://www.sgi.com). While high resolution display walls are available (http://www.visbox.com, NCSA display wall project: http://www.ncsa.uiuc.edu/Divisions/DMV/Vis/Projects/TiledWall/, LCSE PowerWall: http://www.lcse.umn.edu), this type of solution is not practical for disseminating data to researchers around the world due to lack of standardization and high cost. Furthermore, due to the multi-field nature of our simulation, the complete image data from our 
simulation would constitute approximately 200 Gbytes of data and is therefore impractical to distribute over the internet in its raw form, even with Internet III.

(a)

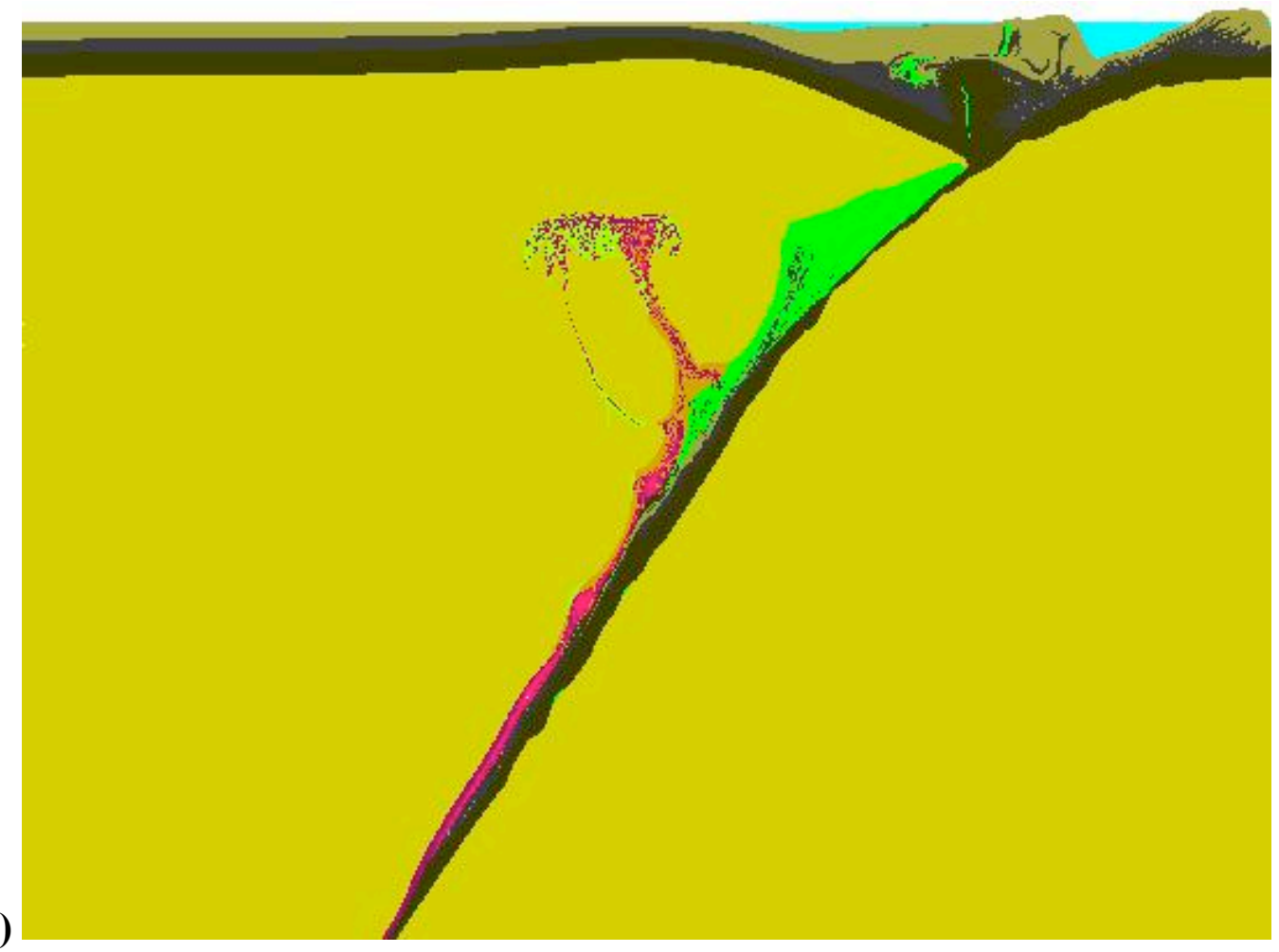

(b)

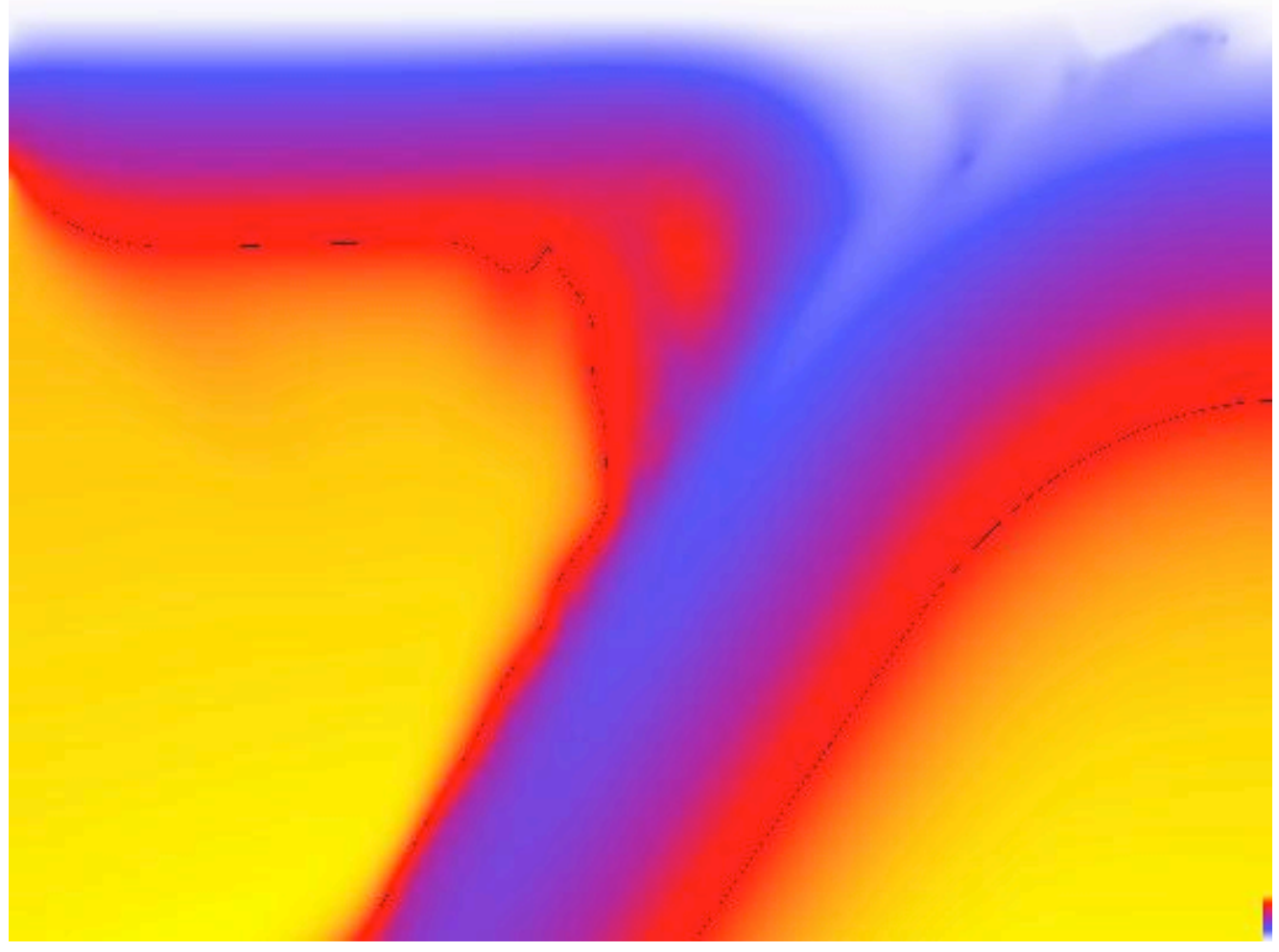


Figure 3. These images are from a run using 100 million markers in a late stage of simulation, and represent: (a) the chemical composition (lithological) field and (b) the temperature field. See Figure 8 for a description of the color code. Chemical composition (lithological) field is represented with resolution about $30 \times 30 \mathrm{~m}$.

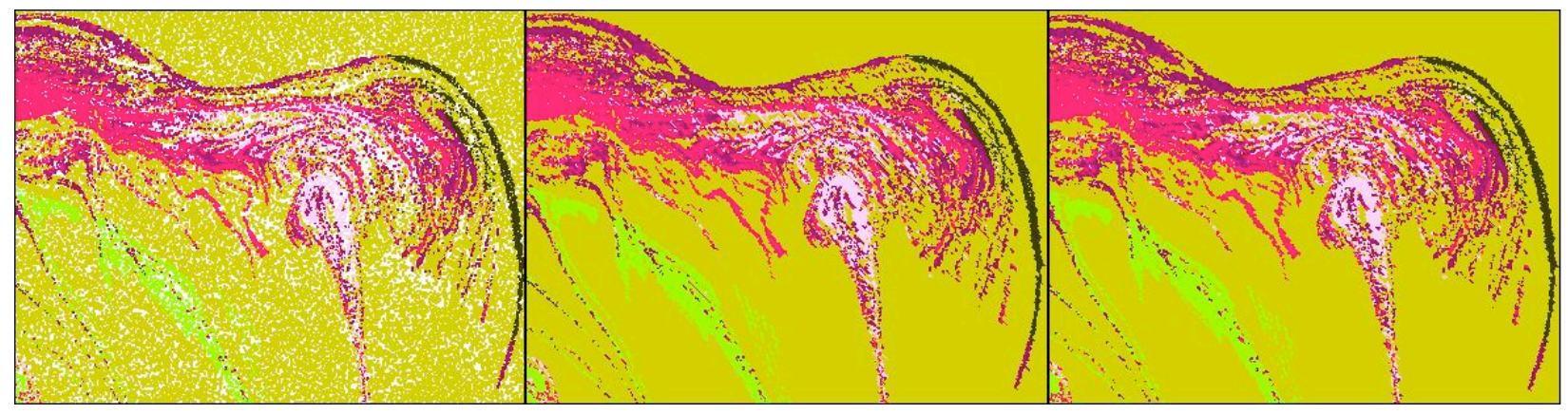

Figure 4. Interpolation of a sparse dataset. The raw data (left) has many voids when mapped onto this area. After processing, the result (right) is more visually pleasing. Please note that these images are exaggerated and normally this amount of data would be mapped onto a smaller image to reduce interpolation and error. See Figure 8 for a description of the color code.

We have addressed this problem of visualization in two ways. In order to view the data locally, we have utilized the University of Minnesota Laboratory for Computational Science and Engineering's PowerWall (http://www.lcse.umn.edu), a high-resolution display wall, to achieve high resolution parallel visualization of multiple fields simultaneously. For remote visualization, we have developed a system for web-based image services which is now incorporated into our WEB-IS visualization toolkit (Garbow et al., 2003; Wang et al., 2003; Yang et al., 2003).

\section{5) WEB-IS Visualization Strategy}

WEB-IS is a recently developed web-service designed specifically for visualizing and interrogating large geophysical, astronomical, and biomedical datasets (Garbow et al., 2001; Wang et al., 2003). It is based on a client-server paradigm, with the server doing all of the processing and storing the data. The WEB-IS image service was designed with several goals in mind. These include hassle-free remote access to data, the ability to view high-resolution data, the ability to view multiple different fields, and low bandwidth requirements. In order to provide a universally compatible interface, we chose to avoid the use of Java entirely. While Java applets and servlets provide robust functionality and excellent real-time user interaction, the burden of installing the Java Virtual Machine rests on the end user. Consequently, we chose to create the 
WEB-IS image service using PHP (PHP: Hypertext Processor, http://www.php.net), a server-side scripting language, and JavaScript, which is integrated into every modern web browser. As a result, our imaging system is accessible through the latest version of any full-featured web browser on any operating system without further software dependencies. Furthermore, the lack of dependence on a Java Virtual Machine allows us to easily access the WEB-IS image service from handheld devices such as the HP Jornada 720 (Boggs and Yuen, 2001) or other handheld devices with Internet connections.

In order to provide high-resolution images on demand, we process our data files prior to a user request. By doing so, we can employ methods of applying color maps and interpolating between markers in areas where marker density is low that are more time-intensive than would be possible for data processing in real-time. The evolution of our interpolation algorithm is illustrated in Fig. 4. Because the interpolation and colormapping can take up to two hours per approximately 30 megapixel image, it is impractical to fully process the raw data into images on demand.

After preprocessing, we are left with a full-resolution, losslessly-compressed PNG image for each property field at each timestep. To minimize bandwidth, WEB-IS dynamically creates lower-resolution versions of these large images for display in a user's web browser. For situations where bandwidth is particularly scarce, WEB-IS incorporates the functionality to display images in the compressed JPEG format, which results in smaller, albeit less detailed, images than those in the default PNG format.

The WEB-IS image service (Fig. 5) incorporates a zoom feature in order to achieve highresolution visualization. The user is first presented with a scaled-down image of the data from our simulation. An area of interest can be selected and our software then automatically creates a large image of the selected region and displays it. An accompanying legend illustrates the relationship of the enlarged region to the entire image. A recently developed feature allows the user to zoom to a specific spatial resolution defined by the physical dimensions of the region of interest. This feature allows geoscientists working remotely to maintain a better understanding of the scale of the simulation thatn would otherwise be possible. The zoom functionality is made possible through cooperation between JavaScript and PHP. When the user clicks and drags to select a region of interest using JavaScript, the web browser transmits information about the region to the server. Using PHP and the GD image library, a new image containing the region of interest is created and sent to the user. This type of functionality has been influential in displaying map 
information over the web, but it is relatively new to the field of multi-component simulation. For example, see the MapServer implementation (http://mapserver.gis.umn.edu), which is used for the Australian Spatial Data Directory (http://asdd.ga.gov.au/servlet/asdd_power).

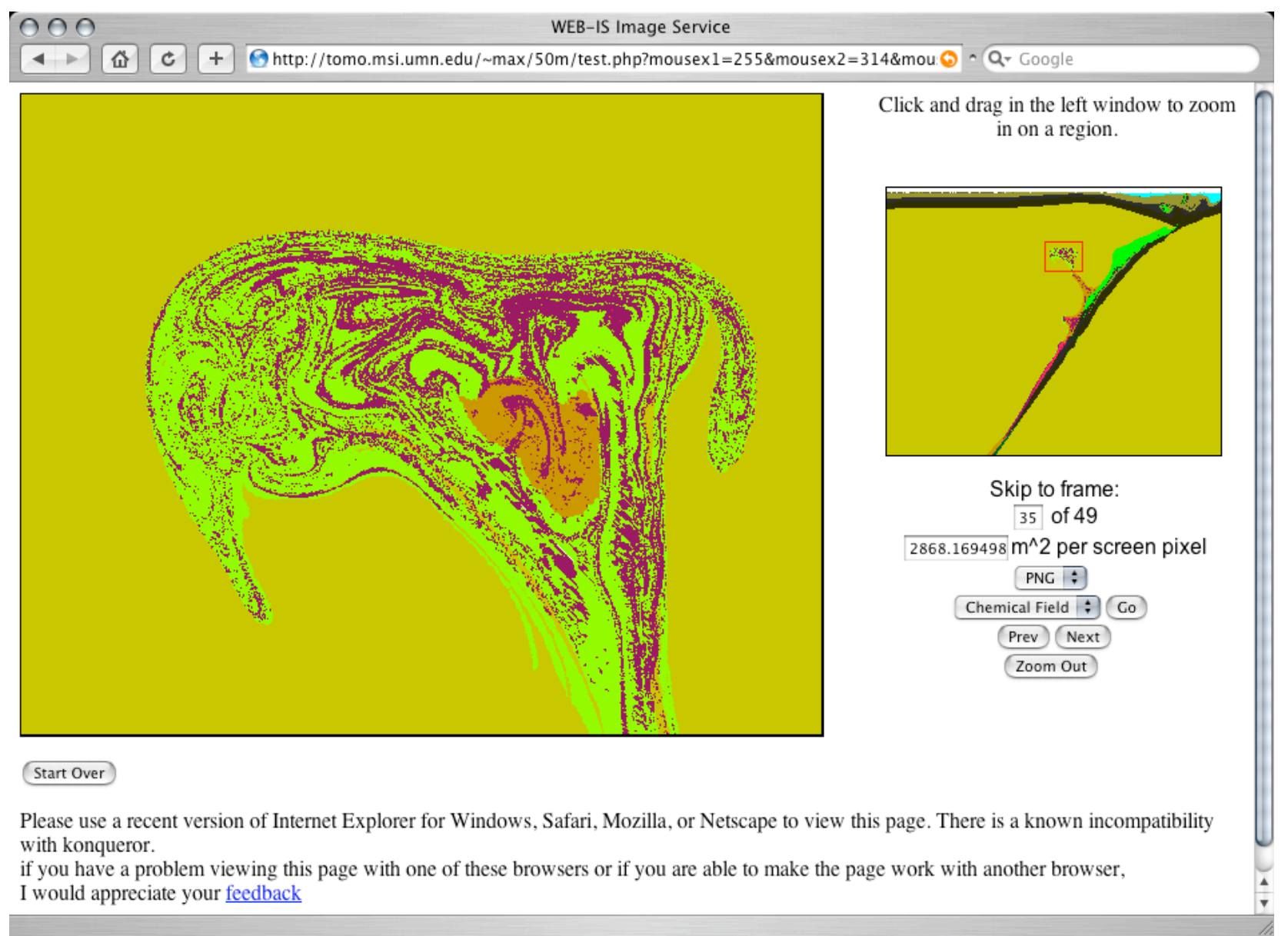

Figure 5. WEB-IS screenshot, presenting the chemical composition (lithological) field. The left box shows a region of interest, while the box on the right shows the entire frame with the region of interest outlined in red. The user controls are also shown. See Figure 8 for a description of the color code.

In addition to the ability to zoom in on an image without loss of detail, WEB-IS provides the user with the ability to select different fields while maintaining a constant timestep and area of interest. This allows the user to see everything going on in the simulation as opposed to just being able to see one component. The insight gained through the development of this multi-aspect view also led to our development of large-scale parallel visualization techniques (Jordan et al., 1996). 


\section{6) Visualization with large-screen display- Power Wall}

Large data sets with many millions of pixels - the same as for high-resolution photographs - can best be viewed on large display devices in order to obtain an entire panoramic view. While the WEB-IS system is clearly our best option for remote visualization and smallscale work on computer monitors, we saw the need for a large-scale alternative to WEB-IS. Through our collaboration with the Laboratory for Computational Science and Engineering (LCSE) at the University of Minnesota, we have gained access to a large display wall that is very well suited for the parallel visualization of subduction zones (Fig 6).

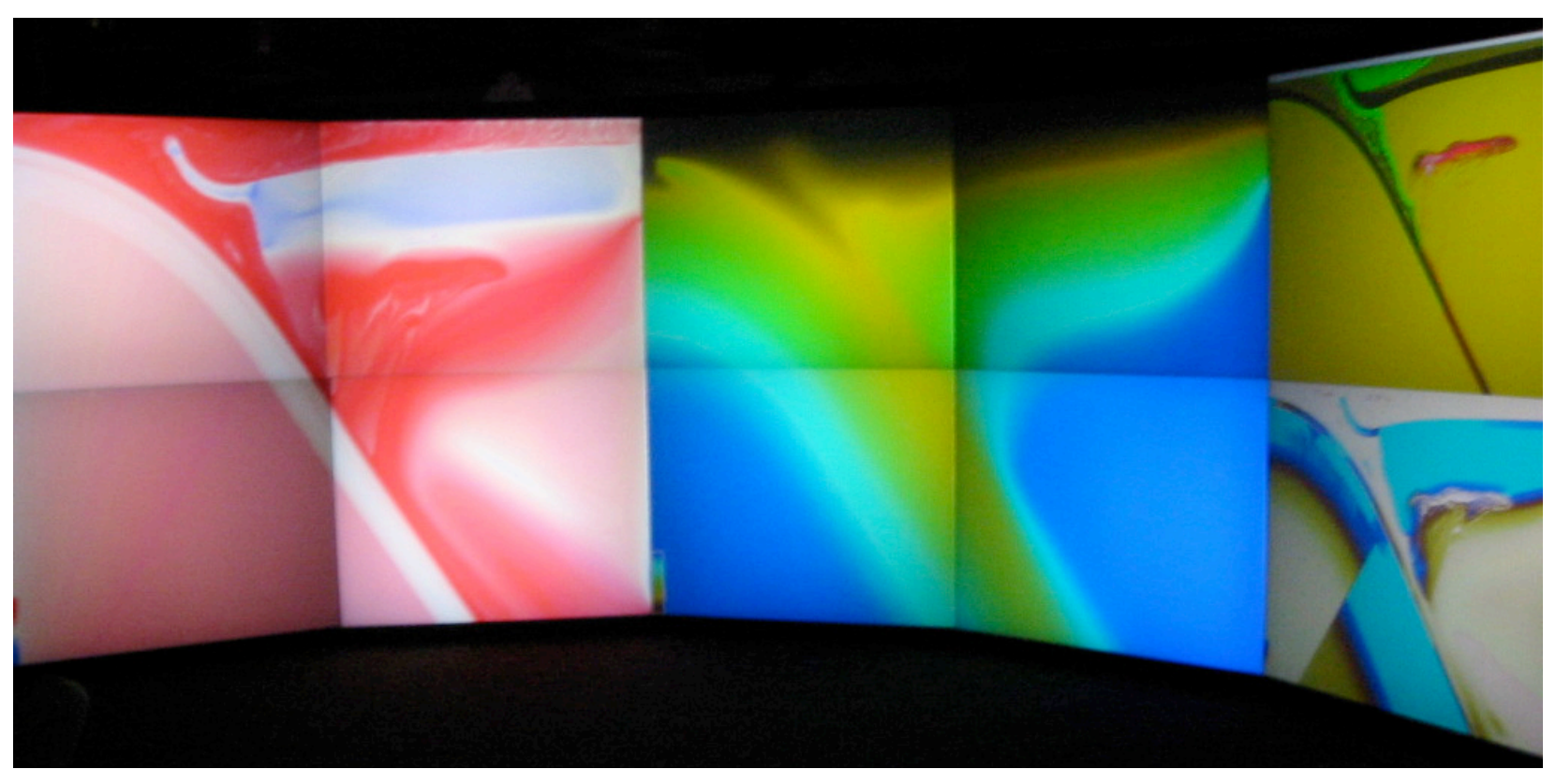

Figure 6. Photograph of the LCSE PowerWall with movies playing on all ten panels. Total size of the PowerWall is 7 feet high and 23 feet wide ( $2 \times 6$ meters).

We note that WEB-IS also enables display on other large-display devices, as one of the authors, Dave Yuen, has done in July 2003 by transmitting the subduction dynamics via Internet from Minnesota to a display wall at Florida State University, about 2200 kilometers away. In the future, presentations involving large data sets will be conducted by using the next generation Internet, whose transmission rate is around $10 \mathrm{Gbits} / \mathrm{sec}$. This method of presentation makes more sense with larger bandwidth in Web communication (Gilder, 2000). Our strategy for 
visualization, as shown in Fig. 7, combines the remote visualization advantages of WEB-IS with the large-scale, high-resolution advantages of devices like the PowerWall.

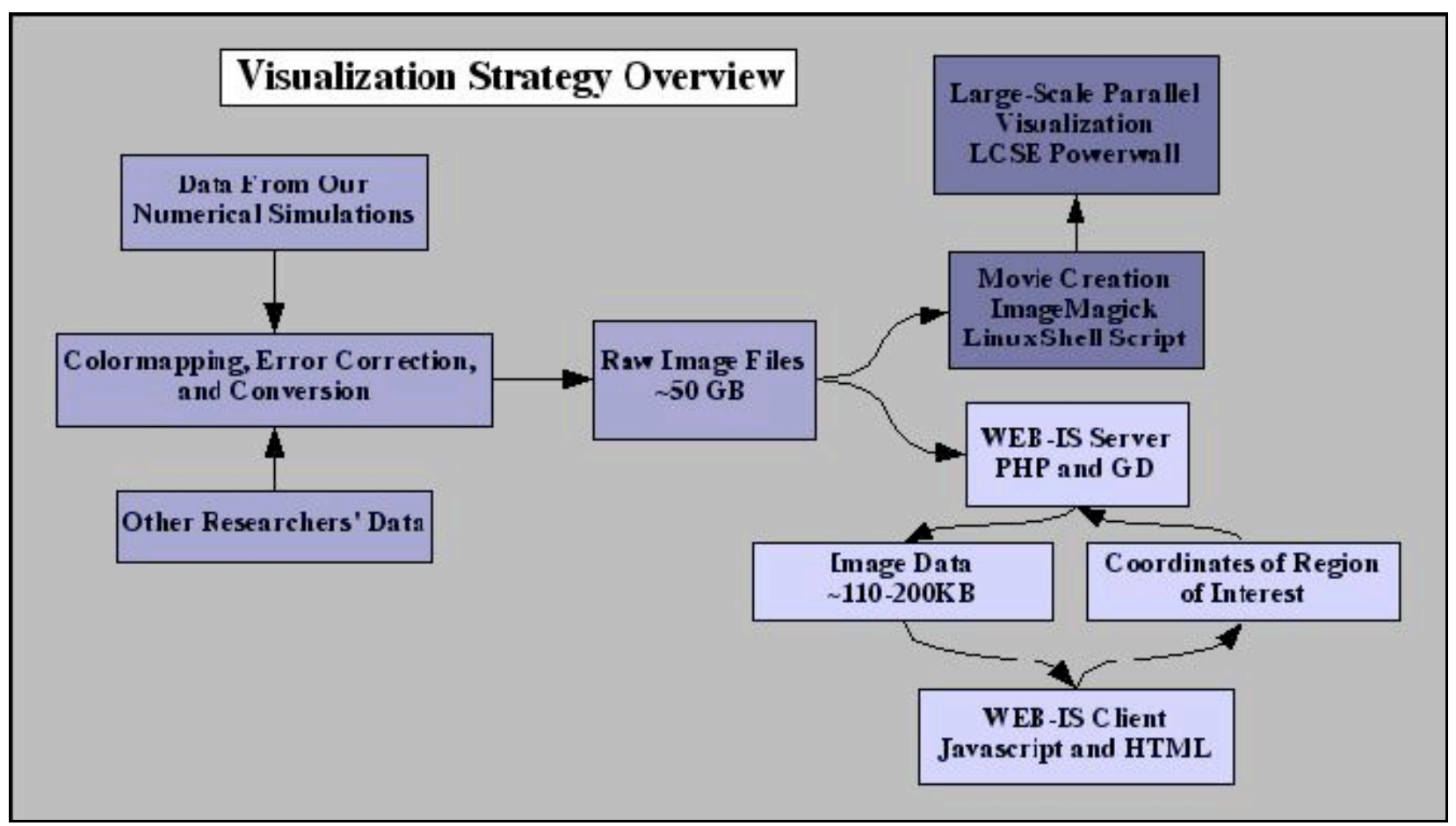

Figure 7. Overview of our visualization strategy, showing progression from raw data to visualization via WEB-IS or the PowerWall.

Located in the Laboratory for Computational Science and Engineering (LCSE: http://www.lcse.umn.edu), headed by Paul Woodward, the PowerWall is a high-performance, high-resolution visualization system for large-scale numerical simulations. An array of ten LCD projectors are arranged to project a seamless image onto a large screen. The 13 million pixels of the PowerWall display, which is 7 feet tall and 23 feet wide ( 2 x 6 meters), allow us to view simulations or other data in unprecedented detail, or to view multiple fields simultaneously, so huge amounts of information can be absorbed by the mind at a single glance. Using the Linux application ImageMagick and a set of custom shell scripts, we are able to convert the same set of images used for WEB-IS into a movie file that can be played on the PowerWall using player software developed by the LCSE team. The PowerWall gives us the flexibility to play up to ten movies simultaneously so that we can examine in great detail the evolution of the subduction zone in multiple components. In Figure 8, we show a movie with 50 frames that portrays the 
temporal development of the chemical composition field for a simulation using 50 million markers.

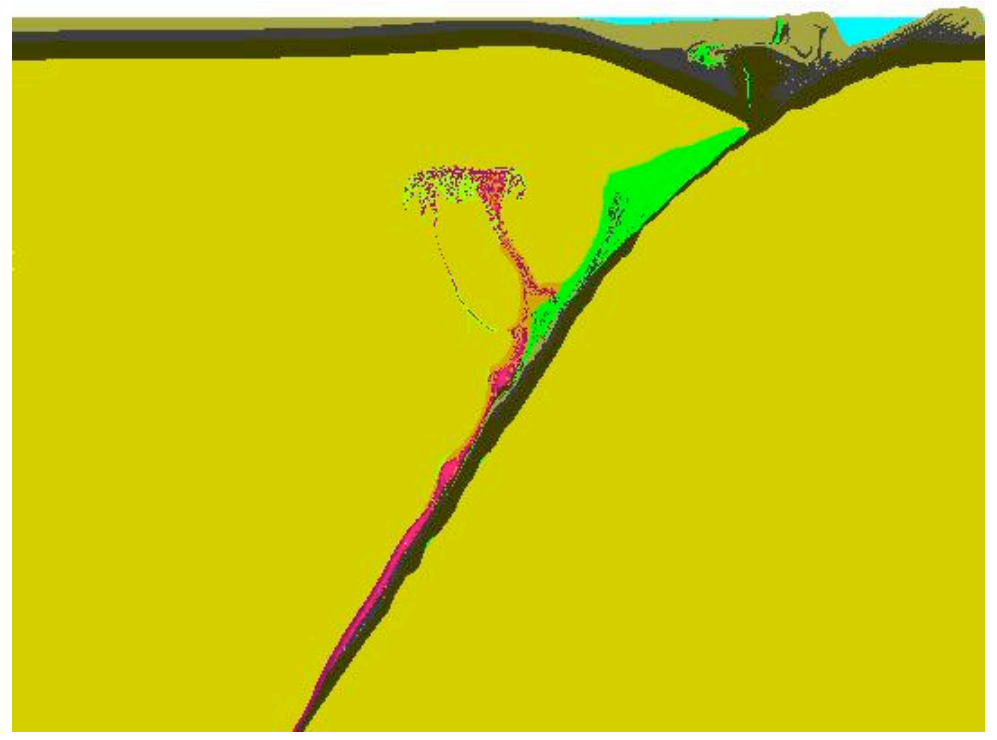

Figure 8. Movie with 50 frames demonstrating the evolution of the chemical composition (lithological) field over time. One frame is shown here, and the full movie is available at http://tomo.msi.umn.edu/ max/movie.html The simulation shown here uses 50 to 100 million markers to

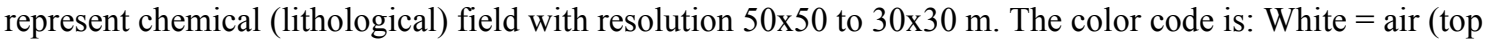
of the model), light blue $=$ sea water (top of the model), grin-brown (both darker and lighter) $=$ solid sedimentary rocks (below sea water and in subduction zone); very dark blue $=$ solid basaltic oceanic crust (upper portion of the oceanic crust immediately below sediments), black = solid gabbroic oceanic crust (lower portion of the oceanic crust), light green $=$ serpentinized mantle (within both the slab and the overriding plate) yellow $=$ unhydrated mantle (both slab and wedge), brown $=$ hydrated unserpentinized (i.e. beyond the stability field of serpentine) mantle, very light green = partially molten hydrated mantle (within plumes); dark magenta $=$ partially molten gabbroic crust (mostly within plumes), light magenta/pink = partially molten basaltic crust (mostly within plumes), very light pink = partially molten sediments (mostly within plumes).

The major advantage of the large-screen strategy is the possibility of very detailed dynamic representation of several (up to 10, optimally 2 to 4 ) fields of physical parameters. The observer can easily change the scale of observation by changing the physical distance from the screen. This mode of visualization provides the optimal set-up for studying both the temporal and causal relationships between large-scale and small-scale phenomena. This can also be exploited as a very powerful and convincing pedagogical tool for students in the geosciences (http://www.geowall.org). 


\section{7) Perspectives and Conclusions}

The use of large numbers of markers allows high-resolution modeling with complex geometry (e.g., Fig. 2). Combined with our recently developed visualization technology, this modeling allows dynamic representation of a subduction zone with a spatial resolution of 10 to 100 meters, the scale at which geologists collect and extrapolate their data. We are, therefore, confident that high resolution modeling is an accessible tool for geologists and that presented numerical results can be tested with geological data on subduction-related geological complexes.

Rapid propagation of "hydrous cold plumes" may wield important control over the spatial distribution of volcanic and seismic activities in regions of subduction (Tamura, 1994; Hall and Kincaid, 2001; Gerya and Yuen, 2003a). Our results also appear to be realistic, when they are compared with the seismic data revealing the complexity of seismicity distributions at subduction zones, especially near the Japanese Trench (Zhao et al., 2002). Negative seismic velocity anomalies found in the Japanese arc constitute a particular feature that might be reinterpreted in light of our results. Rather than regarding them as thermal anomalies (Tamura et al., 2002), we interpret these low-seismic-velocity anomalies (Zhao et al., 2002) near Japan as indicating some traces of water (Jung and Karato, 2001). The generation and propagation of incipient magma chambers above slabs depends strongly on the dynamics imposed by the subduction rate and the thermal constraints posed by the age of the slab and the asthenospheric circulation above the slab (Davies and Stevenson, 1992; Gerya and Yuen, 2003a; Stern, 2002).

Data-centered collaboration on the GRID (Berman et al., 2003) in the emerging new fields of geoinformatics and cyberinfrastructures is expected to revolutionize the geosciences in the next several years. About twenty years ago, Olson et al. (1984) employed 40,000 markers in the studies of mixing dynamics in mantle convection. Today we have reached about 100 million markers. We can then anticipate a decade from now, in 2013, it is not unreasonable to expect the 
bar to be raised to around 10 billion markers! Such a high density of markers would demand entirely new visualization tools and strategies, as well as new approaches to data storage and transmission. Table 1 shows the dependence of the size of required memory versus number of markers for configurations in two dimensions, as described in this paper, and three dimensions, which could be used in the future. Clearly, the increasing number of tracers for this study alone highlights the increasing problems as growing numerical simulations push the limits of available methods of processing, storage, and visualization.

\begin{tabular}{|c|c|c|}
\hline $\begin{array}{c}\text { Number of } \\
\text { markers }\end{array}$ & Memory in 2-D & Memory in 3-D \\
\hline 5 million & 70 Megabytes & 90 Megabytes \\
50 million & 700 Megabytes & 900 Megabytes \\
500 million & 7 Gigabytes & 9 Gigabytes \\
5 billion & 70 Gigabytes & 90 Gigabytes \\
50 billion & 700 Gigabytes & 900 Gigabytes \\
500 billion & 7 Terabytes & 9 Terabytes \\
\hline
\end{tabular}

Table 1. The minimal number of bytes for one marker is calculated using four bytes for each coordinate, four bytes for the temperature, and two bytes for chemical component type. Therefore, one marker uses 14 bytes in two dimensions and 18 bytes in three dimensions. The memory required for various numbers of markers is shown here.

Communications will improve drastically with the Next Generation Internet (Berman et al., 2003). Many information technology experts predict that grids of geographically distributed mass storage across the country along with strategically-located high-performance computers and visualization platforms connected via high-speed $(10 \mathrm{Gbit} / \mathrm{sec}$ to $50 \mathrm{Gbit} / \mathrm{sec})$ networks will allow geoscientists to collaborate seamlessly and remotely. A new era of integrated massive data from distributed, diverse locations will aid earth scientists in discovering new phenomena by datamining and attacking problems across multiple spatio-temporal scales and at the same time traverse over traditional discipline boundaries. The Earth Simulator Center (http://www.es.jamstec.go.jp/esc/eng/) is an example of such an interdisciplinary endeavour, and combines computer science with oceanic, atmospheric, and solid earth simulations. Large output 
files from simulations at Earth Simulator contain a great deal of data, but are difficult to postprocess or visualize in a rapid manner, underscoring the dire need for new visualization tools. As shown in Table 1, 500 billion markers in three dimensions would require nearly 10 terabytes of memory - around the same order of magnitude as the maximum capacity of Earth Simulator and would produce total output on the order of 500 terabytes. These kinds of extreme problems in computational geophysics motivate and shape new strategies in information technology in the geosciences.

Data-centric collaboration of these problems will be undoubtedly carried out on the GRID (Berman et al., 2003), which will revolutionize all of the geosciences in the next several years. In this condition, WEB-IS will gain greater importance for remote visualization as the amount of data in numerical experiments continues to grow. The problems created by large datasets are not unique to the geosciences. Due to its data independence, WEB-IS can be used for remote data query and visualization of biomedical problems, such as the analysis of archived mammograms (http://nscp.upenn.edu/NDMA/), which have a deep social relevance since there is also a need for ultra-large data systems, data-mining, and efficient access to a very large-scale dataset, in the range of petabytes. WEB-IS also has potential applications in astronomy (Murtagh et al., 2002) and in radar interferometry (Fialko and Simons, 2000), where there is a mounting need for viewing data from multiple spectra and viewing high-resolution images involving billions of pixels.

As large display devices like the LCSE PowerWall become cheaper - below the $\$ 5000$ barrier - and also widespread and standardized, high-resolution parallel visualization will gain greater acceptance as the method of choice for viewing multi-component numerical simulations. In this regard, the GeoWall (http://www.geowall.org) can be a good role model for the geosciences.

Because of interdisciplinary activities and feedback from information technology, we see a very bright future lying ahead for visualization in the geosciences, both in large-scale numerical simulations and high-resolution imaging from transmission electron microscopes (TEM), atomic 
force microscopes (AFM), and magnetic resonance imaging (MRI). Technological breakthroughs using liquid crystals and polymers will make electronic paper a viable medium, as well as more portable PowerWalls. All of these developments together will help WEB-IS to fully fuel the rate of progress in visualization in the geosciences.

\section{Acknowledgements}

We thank Yunsong Wang, Gordon Erlebacher, Zack Garbow, Ben Kadlec, Evan F. Bollig, Erik O. D. Sevre, Paul Woodward, David H. Porter, and Michael Bakhos for discussions. This research has been supported by the National Science Foundation. M. Rudolph thanks the Research Experiences for Undergraduates program of the National Science Foundation, in particular. T. Gerya appreciates support by the Russian Foundation of Basic Research grant no. 03-05-64633, by the Alexander von Humboldt Foundation Research Fellowship, and by the German Science Foundation within SFB 526.

\section{References}

Berman F., Fox G., and Hey T. (2003) Grid Computing: Making the Global Infrastructure a Reality. John Wiley and Sons.

Boggs J.B. and Yuen D.A. (2001) Wireless Internet access across Washington Avenue. University of Minnesota Supercomputing Institute Research Report UMSI 2001/135, November 2001. http://www.msi.umn.edu/ boggsj/wireless.html

Davies J.H. and Stevenson D.J. (1992) Physical model of source region of subduction zone volcanics. Journal of Geophysical Research 97: 2037-2070.

Fialko Y. and Simons M. (2000) Deformation and seismicity in the Coso geothermal area, Inyo County, California: observations and modeling using satellite radar interferometry. Journal of Geophysical Research 105: 21781-21793.

Garbow Z.A., Erlebacher G., Yuen D.A., and Boggs, J.M. (2001) Interactive Web-based map: applications to large data sets in the geosciences. Electronic Geosciences, 6.

Garbow Z.A., Yuen D.A., Erlebacher G., Bollig E.F., and Kadlec B.J. (2003) Remote visualization and cluster analysis of 3-D geophysical data over the Internet using offscreen rendering. Visual Geosciences (submitted). 
Gilder G. (2000) Telecosm: How infinite bandwidth will revolutionize our world. New Press. Gerya T.V. and Yuen D.A. (2003a) Rayleigh-Taylor instabilities from hydration and melting propel 'cold plumes' at subduction zones. Earth and Planetary Science Letters 212: $47-62$.

Gerya T.V. and Yuen D.A. (2003b) Characteristics-based marker method with conservative finite-differences schemes for modeling geological flows with strongly variable transport properties. Phys. Earth Planet. Inter. 140: 295-320.

Gerya T.V., Stoeckhert B., and Perchuk A.L. (2002) Exhumation of high-pressure metamorphic rocks in a subduction channel - a numerical simulation. Tectonics 21: 6-1 - 6-19.

Gerya T.V., Yuen D.A., and Sevre E.O.D. (2004) Dynamical causes for incipient magma chambers above slabs. Geology (in press).

Hall P.S. and Kincaid C. (2001) Diapiric flow at subduction zones: A recipe for rapid transport. Science 292: 2472-2475.

Hockney R.W. and Eastwood J.W. (1981) Computer Simulations Using Particles. New York, McGraw-Hill.

Jordan K.E., Yuen D.A., Reuteler D.M., Zhang S., and Haimes R. (1996) Parallel interactive visualization of 3-D mantle convection. IEEE Computational Science and Engineering, Vol. 3, No. 4: 29-37.

Jung, H., and Karato, S. (2001) Water-induced fabric transitions in olivine. Science, 293, $1460-1463$.

Kincaid C. and Hall P.S. (2003) Role of back arc spreading in circulation and melting of subduction zones. Journal of Geophysical Research 108, 7-1 - 7-14.

Marsh B.D. (1979) Island-arc development: some observation, experiments and speculations. Journal of Geology 87: 687-713.

Murtagh F., Starck J.L and Louys M. (2002) Distributed visual information management in astronomy. Computers in Science and Engineering Vol. 4 No. 6: 14-23.

Olson P.L., Yuen D.A., and Balsiger D.S. (1984) Mixing of passive heterogeneities by mantle convection. J. Geophys. Res. 89: 425-436.

Osher S.J. and Fedkiw R. (2002) Level Set Methods and Dynamic Implicit Surfaces. NewYork, Springer Verlag. 
Peacock S.M. (2003) Thermal stucture and metamorphic evolution of subducting slabs. In: Eiler J.M. and Abers G. (eds) The Subduction Factory. AGU Gophysical Monograph, American Geophysical Union, Washington, D. C. (in press).

Ranalli G. (1995) Rheology of the Earth (2nd edition). London, Chapman and Hall.

Sapiro G. (2001) Geometric Partial Differential Equations. Cambridge University Press.

Schmidt M.W. and Poli S. (1998) Experimentally based water budgets for dehydrating slabs and consequences for arc magma generation. Earth Planet. Sci. Lett. 163: 361-379.

Stern R.J. (2002) Subduction zones. Review in Geophysics 40: 3-1 - 3-38.

Tamura, Y. (1994) Genesis of island arc magmas by mantle-derived bimodal magmatism: Evidence from the Shirahama Group, Japan. Journal of Petrology, 35: 619-645.

Tamura Y., Tatsumi Y., Zhao D.P., Kido Y., and Shukuno H. (2002) Hot fingers in the mantle wedge: new insights into magma genesis in subduction zones. Earth and Planetary Science Letters 197: 105-116.

Ten A.A., Yuen D.A., and Podladchikov Yu.Yu. (1999) Visualization and analysis of mixing dynamical properties in convecting systems with different rheologies. Electronic Geosciences 4.

Tovish A., Schuberst G., and Luyendyk B.P. (1978) Mantle flow pressure and the angle of subduction: non-Newtonian cornerflows. J. Geophys. Res. 83: 5892-5898.

Wang Y., Bollig E.F., Kadlec B.J., Garbow Z.A., Erlebacher G., Yuen D.A., Rudolph M., Sevre, E.O.D, and Yang L. (2003) WEB-IS (Integrated System): an overall view. Visual Geosciences (in preparation).

Yang X., Wang Y., Bollig E.F., Kadlec B.J., Garbow Z.A., Yuen D.A., and Erlebacher G. (2003) WEB-IS2: Next generation web services using Amira visualization package. AGU Fall Meeting, Abstract NG11A-0164.

Zhao, D.P., Mishra, O.P., and Sandra, R. (2002) Influence of fluid and magma on earthquakes: Seismological evidence. Physics of the Earth and Planetary Interiors, 132, 249-267. 